Correspondence: Anastasiia Russkikh, Department of Differential Diagnosis and Treatment of Tuberculosis and Associated Infections, Federal State Budgetary Institution "National Medical Research Center of Phthisiopulmonology and Infectious Diseases" of the Ministry of Health of the Russian Federation, Dostoevskogo str. 4, build 2, 127473 Moscow, Russian Federation.

Tel. + 7.985.252-05-95

E-mail: ana-lobach@yandex.ru

Key words: Extensively drug-resistant tuberculosis; culture conversion; median time; bedaquiline; linezolid.

Acknowledgements: The authors thank the Federal State Budgetary Institution "National Medical Research Center of Phthisiopulmonology and Infectious Diseases" of the Ministry of Health of the Russian Federation for defining research questions and providing data for this study, and the secretariat of the European TB Research Initiative (ERI$\mathrm{TB}$ ) at the WHO Regional Office for Europe for organizing the Structured Operational Research Training (SORT-TB) for eastern European countries supported by the USAID-WHO regional partnership project to End TB in eastern Europe. SORT-TB curriculum was an adaptation of the UNICEF/UNDP/World Bank/WHO Special Programme for Research and Training in Tropical Diseases (TDR) SORT IT course (https://www.who.int/tdr/capacity/strengthening/ sort/en/) to the eastern European context.

Contributions: AR, OK, AS, JA, AY, MD, IV, conceived the study aims. All authors participated in the design, discussion of the results interpretation, read, edited, and agreed with the decision to submit the final version of the paper. AR, data collection, reference review; AR, AS, YS, analysis design and execution; AR, OK, manuscript first draft; AS, JA, AY, $\mathrm{MD}$, IV, substantial revisions to the initial version of the manuscript; AR, AS, IV are staff members of the Federal State Budgetary Institution "National Medical Research Center of Phthisiopulmonology and Infectious Diseases" of the Ministry of Health of the Russian Federation.

Conflict of interest: The authors declare no conflict of interest.

Funding: None.

Ethics approval: Permission to access the data was obtained from the Federal State Budgetary Institution "National Medical Research Center of Phthisiopulmonology and Infectious Diseases" of the Ministry of Health of the Russian Federation. Ethics approval was obtained from the Local Ethics Committee of the Federal State Budgetary Institution "National Medical Research Center of Phthisiopulmonology and Infectious Diseases" of the Ministry of Health of the Russian Federation (Extract No. 31/Protocol No. 82/25.12.2019). Exemption from an ethics review was also received from the World Health Organization Research Ethics Review Committee based in Geneva, Switzerland (ERC.0003319/11.03.2020). A waiver of informed consent was granted by ethics review bodies, as the study collected and analysed de-identified routine recording and reporting data.

Disclaimer: The authors alone are responsible for the views expressed in this publication and they do not necessarily represent the decisions or policies of the World Health Organization.

Received for publication: 10 July 2020.

Accepted for publication: 12 August 2020.

OWorld Health Organization 2021.

All rights reserved. The WHO Regional Office for Europe has granted the Publisher permission for the reproduction of this article.

Licensee PAGEPress, Italy

Monaldi Archives for Chest Disease 2021; 91:1678

doi: 10.4081/monaldi.2021.1678

Open access statement: In accordance with WHO's open-access publication policy for all work funded by WHO or authored/co-authored by WHO staff members, the WHO retains the copyright of this publication through a Creative Commons Attribution IGO licence (http://creativecommons.org/licenses/by/3.0/igo/legalcode) which permits unrestricted use, distribution and reproduction in any medium provided the original work is properly cited.

\section{Factors associated with culture conversion among adults treated for pulmonary extensively drug-resistant tuberculosis during 2018-2019 in the Russian Federation: an observational cohort study}

\author{
Anastasiia Russkikh ${ }^{1}$, Oleksandr Korotych ${ }^{2}$, \\ Yuliia Sereda ${ }^{2}$, Anastasia Samoilova ${ }^{1}$, \\ Jay Achar ${ }^{3,4}$, Askar Yedilbayev², Masoud Dara², \\ Irina Vasilyeva ${ }^{1}$
}

${ }^{1}$ Federal State Budgetary Institution “National Medical Research Center of Phthisiopulmonology and Infectious Diseases" of the Ministry of Health of the Russian Federation, Moscow, Russian Federation; ${ }^{2}$ World Health Organization, Regional Office for Europe, Copenhagen, Denmark; ${ }^{3}$ Department of Global Public Health, Karolinska Institutet, Stockholm, Sweden; ${ }^{4}$ Department of Medical Epidemiology and Biostatistics, Karolinska Institutet, Stockholm, Sweden

\section{Abstract}

Treatment outcomes for multidrug/rifampicin-resistant tuberculosis (MDR/RR-TB) and extensively drug-resistant tuberculosis (XDR-TB) remain poor across the globe and in the Russian Federation. Treatment of XDR-TB is challenging for programmes and patients often resulting in low success rates and onward transmission of drug-resistant strains. Analysis of factors affecting culture conversion rate among XDR-TB patients may serve as a basis for optimization of treatment regimens.

We conducted a retrospective cohort study using health records from 54 patients with pulmonary XDR-TB treated at a tertiary level facility in the Russian Federation. The study population included adult patients with culture-positive pulmonary XDR-TB who started treatment between 1 January 2018-30 June 2019. Culture conversion was defined as two consecutive negative cultures, collected at least 30 days apart. The date of sputum culture conversion was taken from the first of two consecutive negative sputum cultures fulfilling these criteria. We measured time to culture conversion using cumulative incidence functions accounting for competing risks and applied binary cause-specific Cox regressions to assess associated factors. Sputum culture conversion was recorded for $43(79.6 \%)$ patients. Median time to culture conversion adjusted for competing risk of loss to follow up was 4 months [95\% confidence interval (CI): 2-5]. The number of patients who had culture converted by treatment months 2,4 , and 6 were 12 (22\%), 29 (54\%) and 38 (70\%) respectively. In unadjusted analysis, positive baseline sputum smear microscopy [hazard ratio (HR): $0.34,95 \%$ CI: $0.18-0.66$; $\mathrm{p}=0.001$ ), hepatitis C (HR: 0.35 , 
95\% CI: 0.14-0.89; $\mathrm{p}=0.023$ ], and human immunodeficiency virus (HR: $0.3095 \%$, CI: $0.09-0.97$; $\mathrm{p}=0.045$ ), and receipt of fewer than 4 effective drugs in the treatment regimen (HR: $0.13,95 \% \mathrm{CI}$ : $0.03-0.60 ; p=0.009)$ were associated with delayed culture conversion. When compared to their combined use, patients receiving regimens with bedaquiline only (HR: $0.12,95 \% \mathrm{CI}$ : $0.03-0.49$; $\mathrm{p}=0.003$ ) or linezolid only (HR: $0.21,95 \% \mathrm{CI}$ : 0.06-0.69; $\mathrm{p}=0.010$ ) were less likely to achieve timely culture conversion. Factors delaying sputum culture conversion should be considered in the management of patients with XDR-TB and considered by clinicians for regimen design and treatment strategies. Our study outlines the importance of simultaneous inclusion of bedaquiline and linezolid in treatment regimens for patients with XDR-TB to reduce time to sputum conversion and increase treatment success.

\section{Introduction}

Globally, tuberculosis (TB) accounts for more deaths than any other single infectious disease. In 2018, an estimated 1.45 million people died due to TB [1]. In 2017, by endorsing the Moscow Declaration to End TB at the World Health Organization global conference in the Russian Federation, Member States committed to increase multisectoral action and accountability in the response towards ending TB by 2030 [2]. Drug-resistant TB, which remains a significant threat to public health represents one of the main obstacles in achieving the goals of the End Strategy $[1,3,4]$. Timely and accurate detection of drug resistance is essential to provide effective treatment. However, in some settings, due to programmatic and patient-related factors, detection of drug-resistance may be delayed. Historically, recommended treatment for XDR-TB was long, toxic and ineffective. In recent years, newer, more effective drugs including bedaquiline, linezolid and delamanid are being used in more countries and are now being combined in short, effective treatment regimens [5]. The increasing prevalence of drugresistant strains of Mycobacterium tuberculosis (MTB) complicates ending tuberculosis [6].

Global and Russian Federation treatment outcomes for multidrug/rifampicin-resistant tuberculosis (MDR/RR-TB) and extensively drug-resistant tuberculosis (XDR-TB) remain sub-optimal and far below regional targets for MDR-TB (75\% treatment success rate among the MDR/RR-TB patients by 2020) [4,7]. $\mathrm{MDR} / \mathrm{RR}-\mathrm{TB}$ is defined as the resistance to at least rifampicin or both isoniazid and rifampicin, while MTB strains exhibiting additional resistance to fluoroquinolones and a second-line injectable agent are classified as XDR-TB [8-10]. In 2019 the treatment success for XDR-TB patients starting treatment in 2017 in the Russian Federation was $38 \%$ compared to the global rate of only $39 \%$ [1].

With the currently available tools, treatment of XDR-TB is complicated, often resulting in low success rates and subsequent amplification of resistance to remaining second-line drugs [4]. One of the potential causes of amplification of resistance is inadequate early treatment regimens or monotherapy due to insufficient numbers of effective drugs [11], which is associated with low treatment success [12].

Ongoing randomized controlled trials are investigating the efficacy of regimens containing recently discovered and repurposed anti-TB drugs (e.g., pretomanid, bedaquiline, linezolid, clofazimine and delamanid) with shorter treatment durations [13-15]. However, data on the performance of newer drugs amongst patients with XDR-TB, particularly in combination, is limited [16].

Since access to highly effective MDR/RR-TB treatment, including XDR-TB treatment, is a core component of TB control measures, analysis of factors affecting treatment outcomes of MDR/RR-TB and XDR-TB may serve as a basis for optimization of treatment regimens, development and introduction of new combinations of TB drug and antibiotics with anti-TB effect into clinical practice. A number of studies suggest that early culture conversion can serve as an acceptable predictive marker for MDR-TB and XDR-TB treatment success [17-19].

The effective management of M/XDR-TB in Russian Federation is a one of priority areas of work for the Federal State Budgetary Institution "National Medical Research Center of Phthisiopulmonology and Infectious Diseases" of the Ministry of Health of the Russian Federation. The institution is in charge of the National TB Programme. Within this study we aimed to describe the factors associated with sputum culture conversion as a favorable interim treatment outcome among adult pulmonary XDR-TB patients who started treatment between 1 January 2018 and 30 June 2019 at the NMRC PhPl.

\section{Materials and Methods}

\section{Study design}

We conducted a retrospective cohort study using health records from adult patients ( $\geq 18$ years) with pulmonary XDR-TB. Patient records were reviewed during the first 6 months of treatment up to culture conversion or declaration of unfavourable outcome, such as death or loss to follow up. If the first negative culture's result after the baseline was received at the $6^{\text {th }}$ month, patients were followed for two additional months. Available data up to 24 February 2020 were included in the study.

\section{Setting}

The study was conducted in $\mathrm{NMRC} \mathrm{PhPl}$, a tertiary referral facility and leading TB institution based in Moscow, which provides inpatient and outpatient care for TB and human Immunodeficiency Virus (HIV) to patients referred from different regions of Russian Federation. Specialists at the facility consult patients in outpatient settings and refer them for diagnostic interventions, when necessary. NMRC PhPl also provides telemedicine consultations for regional clinics. The decision to admit a patient to NMRC PhPl is based on a referral from a regional clinic and the decision of the NMRC PhPl expert committee. The NMRC PhPl has a specialized Department for the treatment of M/XDR-TB. Over $300 \mathrm{M} / \mathrm{XDR}-\mathrm{TB}$ patients are treated at the Department annually. The Department has 70 inpatient beds for adult patients ( $\geq 18$ years) from all regions of the Russian Federation, as well as from other countries. Treatment decisions are taken by the NMRC PhPl expert committee and are based on national guidelines [9].

In Russia all WHO-recommended Group A, B and C drugs for longer regimens of DR-TB are available, except clofazimine, which remains unregistered. Delamanid received National regulatory authority approval in May 2020 and was not available to patients during the study period. All patients were hospitalized for treatment initiation with discharge primarily influenced by culture conversion status. Treatment in the hospital was directly observed by trained medical staff. Diagnosis of TB at NMRC PhPl was done at local/national reference laboratory and confirmed by approved molecular diagnostics in addition to liquid culture testing (BACTEC ${ }^{\text {TM }}$ Mycobacterial Growth Indicator Tube ${ }^{\text {TM }}$ 960) and solid Lowenstein-Jensen media. Baseline drug susceptibility testing (DST) to all 1st and 2nd line TB drugs was performed on all 
diagnostic specimens [20]. During treatment, monthly sputum samples were collected for culture on solid media.

\section{Study population}

We included all consecutive adults ( $\geq 18$ years) with sputum culture-positive laboratory confirmed pulmonary XDR-TB who started treatment at NMRC PhPl between $1^{\text {st }}$ January $2018-30^{\text {th }}$ June 2019.

\section{Data variables}

Variables included patient demographic, behavioural and social characteristics, data on TB diagnostics and treatment, such as individual drugs in the regimen (any duration of uptake), number of effective drugs in the regimen and number of days without anti-TB medication during the first 6 months of treatment. Effective drug was defined as drug to which susceptibility of MTB was either confirmed by a reliable drug-resistance testing or assumed based on patient's treatment history or results of drugresistance studies (new TB drugs or antibiotics with anti-TB activity; drugs that were not previously part of ineffective regimen for a patient; high susceptibility of MTB strains for this drugs in patients with a similar TB history according to results of population drug-resistance studies) [9]. In this study, the day without antiTB medication was defined as missing of all drugs in the regimen during the day. All patients were hospitalized throughout the follow-up period and received medication once a day. Culture conversion was defined as two consecutive negative cultures, collected at least 30 days apart. The specimen collection date of the first negative culture was used as the day of conversion. Follow-up time was recorded in days from the date of XDR-TB treatment initiation to culture conversion, death or loss to follow up. Patients were censored after 8 months of follow-up or at the end of the study $\left(24^{\text {th }}\right.$ February 2020). When a patient died or was lost after culture conversion during the study period, they were considered as converted. Laboratory data included sputum smear microscopy, date of sputum samples collection and solid media culture results for MTB for all available samples. Testing for HIV and viral hepatitis $\mathrm{C}$ was performed prior to therapy.

\section{Data collection and analysis}

Data were extracted from individual patient paper records and local laboratory database and entered into an EpiData database. In order to ensure data quality, $10 \%$ of records were double entered. Data validation rules were designed and deployed to check accuracy and consistency of data. We summarized demographic, behavioural and clinical characteristics of the study participants at baseline with frequencies and proportions (for categorical variables) and mean (and standard deviation) or median (and interquartile range) for continuous variables, as appropriate.

We measured time to culture conversion using cumulative incidence function to account for competing risk of loss to follow up and assessed the factors associated with culture conversion. We applied binary cause-specific Cox regressions to assess factors associated with time to culture conversion in the presence of competing events. Sample size did not allow to conduct an adjusted analysis. Levels of significance was set at $5 \% \quad(\mathrm{p}<0.05)$. Proportional hazard assumption was tested by using the Schoenfeld residuals. All selected covariates met the assumption. Analysis was done using R, version 3.5.2 software ( ${ }^{\circ} \mathrm{R}$ Foundation for Statistical Computing, 2016).

Table 1. Characteristics of adult pulmonary XDR-TB patients who started treatment between January 2018 and June 2019 at the Federal State Budgetary Institution "National Medical Research Center of Phthisiopulmonology and Infectious Diseases" of the Ministry of Health of the Russian Federation ( $n=54)$.

\begin{tabular}{|c|c|c|c|}
\hline & & $\mathrm{n}$ & $(\%)$ \\
\hline Demographic and behaviour characteristics & Total & 54 & (100) \\
\hline $\begin{array}{l}\text { Sex } \\
\text { Male } \\
\text { Female }\end{array}$ & & $\begin{array}{l}30 \\
24\end{array}$ & $\begin{array}{l}(56) \\
(44)\end{array}$ \\
\hline Age, years & & & \\
\hline $\begin{array}{l}\text { Mean age (standard deviation) } \\
18-34 \\
35-44 \\
45-63\end{array}$ & & $\begin{array}{l}35.6 \\
24 \\
20 \\
10\end{array}$ & $\begin{array}{l}(10) \\
(44) \\
(37) \\
(19)\end{array}$ \\
\hline $\begin{array}{l}\text { Employment } \\
\text { Employed } \\
\text { Unemployed } \\
\end{array}$ & & $\begin{array}{l}18 \\
36\end{array}$ & $\begin{array}{l}(33) \\
(67)\end{array}$ \\
\hline $\begin{array}{l}\text { History of incarceration } \\
\text { Yes } \\
\text { No } \\
\end{array}$ & & $\begin{array}{c}4 \\
50\end{array}$ & $\begin{array}{l}(7) \\
(93)\end{array}$ \\
\hline $\begin{array}{l}\text { Current smoker } \\
\text { Yes } \\
\text { No } \\
\text { Not recorded } \\
\end{array}$ & & $\begin{array}{l}28 \\
25 \\
1\end{array}$ & $\begin{array}{l}(52) \\
(46) \\
(2)\end{array}$ \\
\hline $\begin{array}{l}\text { Harmful use of alcohol } \\
\text { Yes } \\
\text { No }\end{array}$ & & $\begin{array}{c}6 \\
48\end{array}$ & $\begin{array}{l}(11) \\
(89)\end{array}$ \\
\hline $\begin{array}{l}\text { Drug abuse } \\
\text { Yes } \\
\text { No }\end{array}$ & & $\begin{array}{c}1 \\
53\end{array}$ & $\begin{array}{c}(2) \\
(98)\end{array}$ \\
\hline
\end{tabular}




\section{Results}

\section{Baseline characteristics}

Between the $1^{\text {st }}$ January 2018 and the $30^{\text {th }}$ June 2019,248 adult patients diagnosed with XDR-TB were admitted to NMRC PhPl specialized Department for the treatment, of which 54 (22\%) were culture positive at baseline and were included in this study. Median follow up time was 6.9 months [interquartile range (IQR): 6.0-7.6]. Mean age of our cohort was 36 (standard deviation \pm 10 ) years and more than half $(30 / 54,56 \%)$ were male (Table 1$)$. Few patients reported harmful use of alcohol $(6 / 54,11 \%)$, but half were current

Table 1. Continued from previous page.

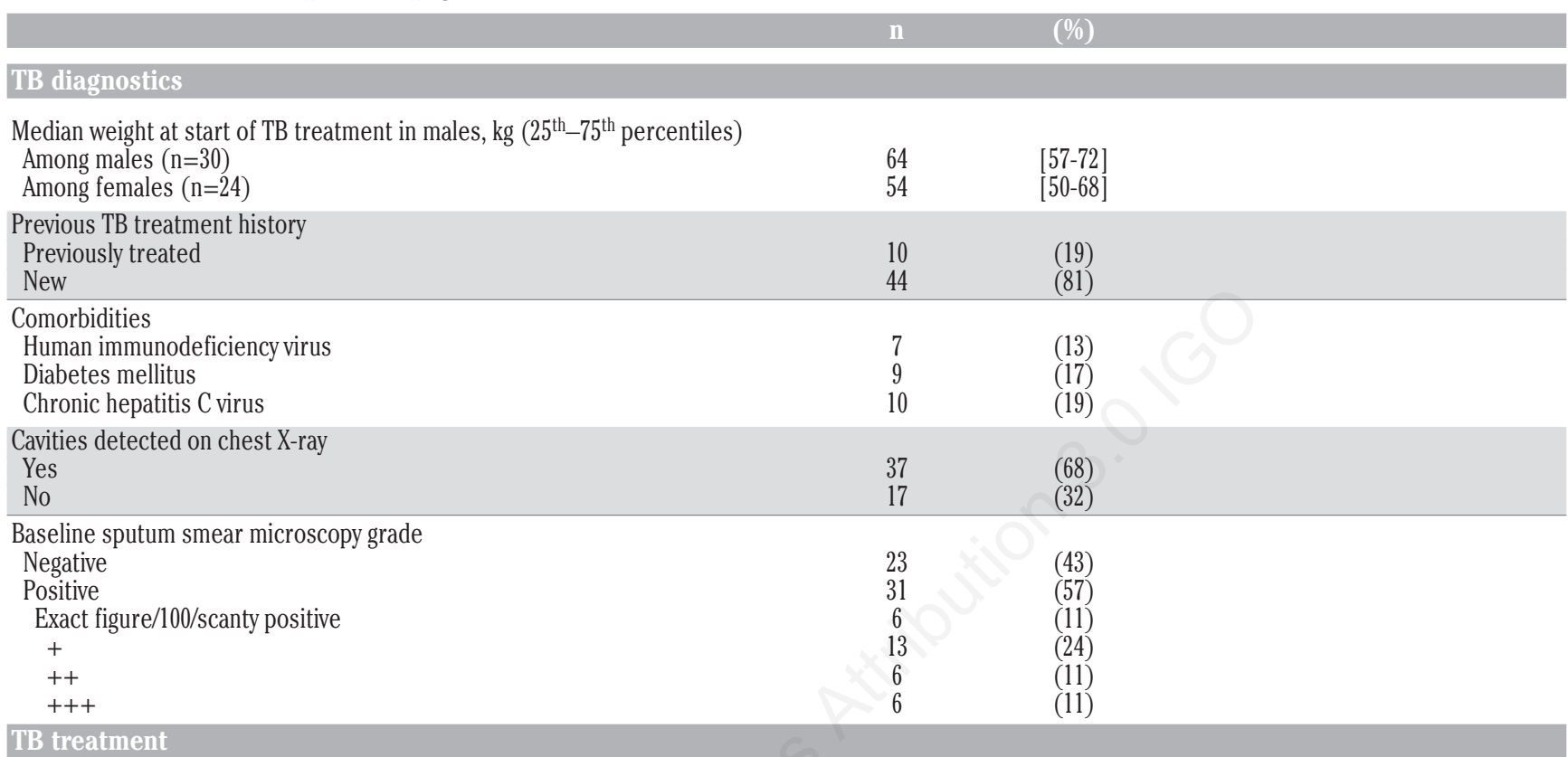

Drugs in the treatment regimen

Bedaquiline

Linezolid

Levofloxacin

Moxifloxacin

Sparfloxacin

Cycloserine

Terizidone

Amikacin

Kanamycin

Capreomycin

Prothionamide

Ethambutol

Meropenem

Imipenem-cilastatin

p-aminosalicylic acid

Pyrazinamide

Number of anti-TB drugs in the treatment regimen

4
5

6

7

8-10

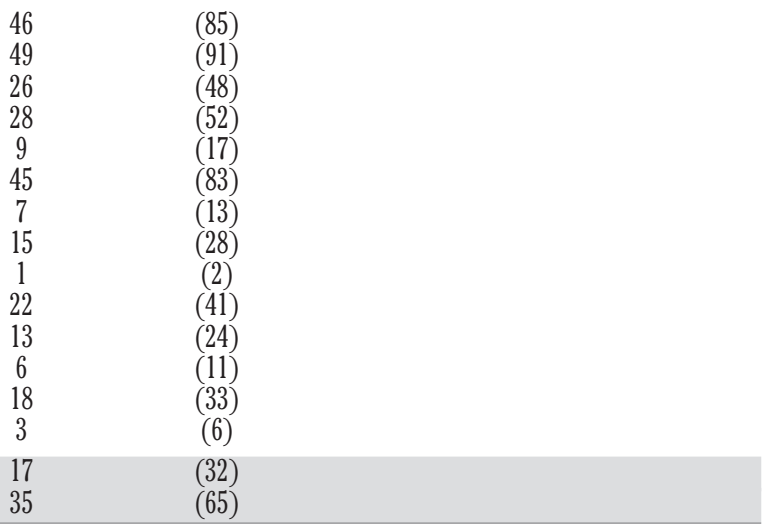

Number of effective drugs in the treatment regimen ${ }^{\circ}$

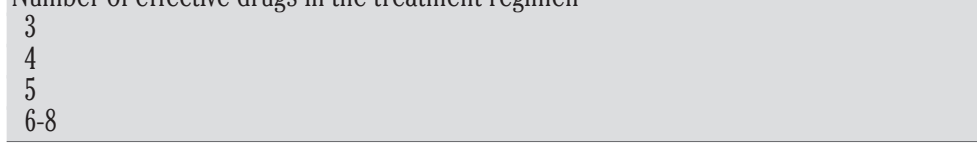

At least one day without anti-TB medication for the first 6 months of treatment

Yes

No

Not recorded

$\begin{array}{cc}1 & (2) \\ 11 & (20) \\ 25 & (46) \\ 11 & (20) \\ 6 & (11)\end{array}$

-Effective drugs are defined: MTB drug sensitivity is confirmed based on the Mycobacterium tuberculosis drug susceptibility test (DST); MTB drug sensitivity is assumed (new TB drugs or antibiotics with anti-TB activity; drugs that were not previously part of an ineffective regimen for this patient; high susceptibility of MTB strains for these drugs in patients with a similar TB history according to results of population drugresistance studies). TB, tuberculosis; XDR, extensively drug-resistant. 
smokers $(28 / 54 ; 52 \%)$. The majority of patients were newly diagnosed $(44 / 54 ; 81 \%)$ and sputum smear microscopy positive $(31 / 54$; $57 \%)$. Seven of 54 patients (13\%) were living with HIV, $10(19 \%)$ had chronic hepatitis $\mathrm{C}$, and $9(17 \%)$ had diabetes mellitus. Baseline chest X-ray identified pulmonary cavities in the majority of patients $(37 / 54 ; 68 \%)$.

The most common drugs included in treatment regimens during the study period were linezolid $(49 / 54 ; 91 \%)$, bedaquiline (46/54; 85\%), cycloserine $(45 / 54 ; 83 \%)$ and fluoroquinolones (levofloxacin: 26/54, 48\%; moxifloxacin: 28/54, 52\%; sitafloxacin: $9 / 54,17 \%$ ). All patients received at least 4 drugs in their regimen. Over a quarter of patients $(15 / 54 ; 28 \%)$ had at least one day without anti-TB medication during the first 6 months of treatment. Among these 15 patients, number of days without anti-TB medication ranged from 3 to 59 with a median of 14 (IQR: 7-22). During the first 6 months of treatment, 42 patients (78\%) were on anti-TB medication $\geq 95 \%$ of days, 6 patients $(11 \%)-90-94 \%$ of days and 4 patients $(7 \%)-67-89 \%$ of days. A minority of patients $(7 / 54$;
$13 \%$ ) received 3 effective drugs in their treatment regimen, while the majority received 5 effective drugs (29/54; 54\%).

\section{Time to culture conversion}

Sputum culture conversion was detected in 43 (79.6\%) patients during follow-up. Median time to culture conversion adjusted for competing risk of loss to follow up was 4.0 months (95\% CI: $2-$ $5)$. The number of patients who had culture converted by treatment months 2, 4, and 6 were 12 (22\%), 29 (54\%) and 38 (70\%) respectively. Loss to follow up was registered for $9 \%(5 / 54)$ of patients (Figure 1).

\section{Factors associated with culture conversion}

In unadjusted analysis, positive baseline sputum smear microscopy (HR: 0.34, 95\% CI: 0.18-0.66; $\mathrm{p}=0.001$ ), hepatitis C (HR: 0.35 , 95\% CI: $0.14-0.89 ; \mathrm{p}=0.023$ ) and HIV comorbidities (HR: $0.30,95 \%$ CI: 0.09-0.97; $\mathrm{p}=0.045$ ), and receipt of fewer than 4 effective drugs in the treatment regimen (HR: $0.13,95 \%$ CI: $0.03-$

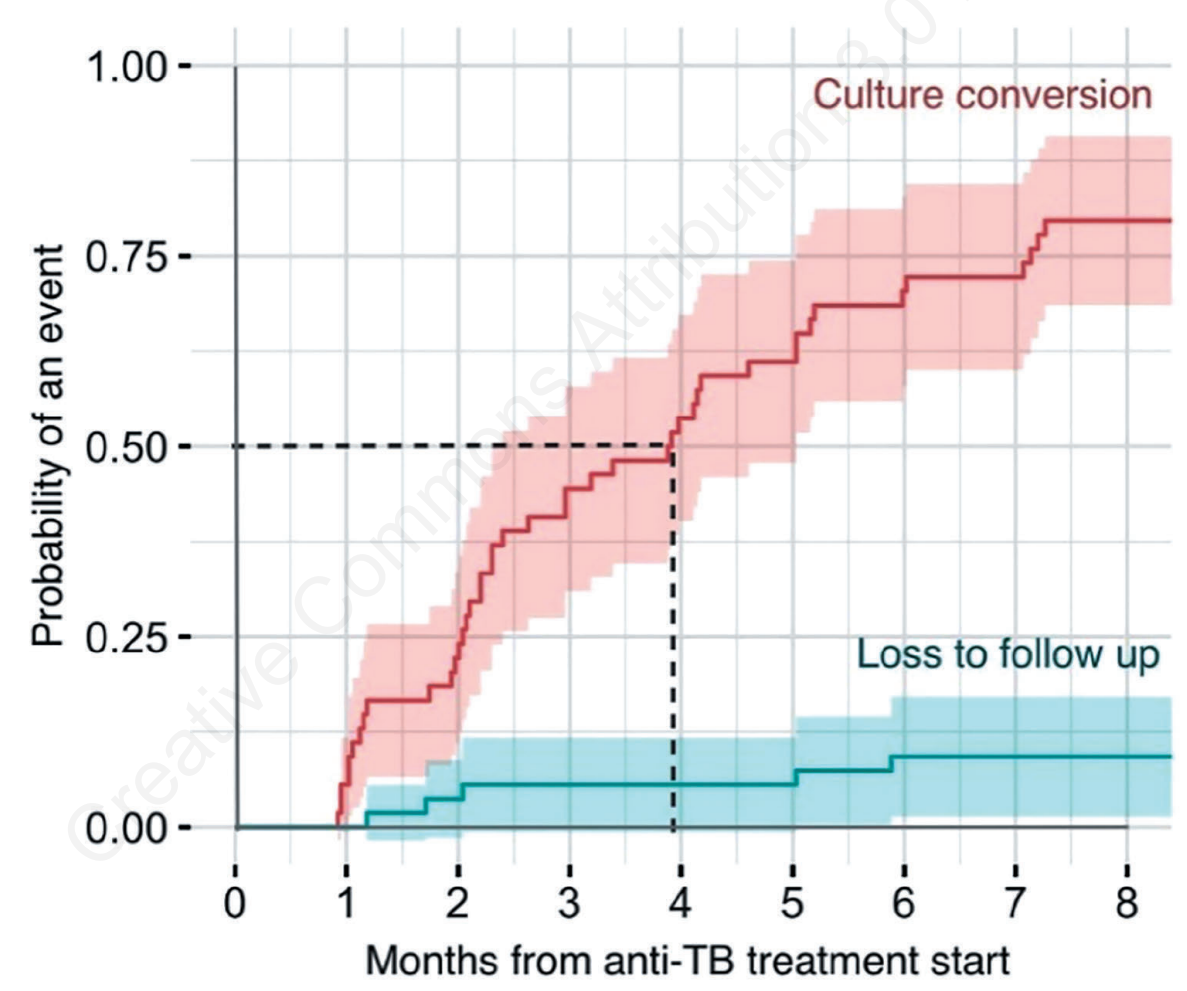

\begin{tabular}{|c|c|c|c|c|c|c|c|c|c|}
\hline Month & 0 & 1 & 2 & 3 & 4 & 5 & 6 & 7 & 8 \\
\hline At risk, $\mathrm{n}$ & 54 & 51 & 40 & 27 & 22 & 18 & 5 & 4 & 0 \\
\hline $\begin{array}{l}\text { Conversions } \\
\text { number }\end{array}$ & - & 3 & 9 & 12 & 5 & 4 & 5 & 1 & 4 \\
\hline cumulative \% & - & 6 & 22 & 44 & 54 & 61 & 70 & 72 & 80 \\
\hline Loss to follow up, $n$ & _- & _ & 2 & 1 & _- & - & 2 & - & _ \\
\hline
\end{tabular}

Figure 1. Cumulative incidence curves of time to culture conversion and loss to follow up among adult pulmonary XDR-TB patients who started treatment between 1 January 2018 - 30 June 2019 at the Federal State Budgetary Institution "National Medical Research Center of Phthisiopulmonology and Infectious Diseases" of the Ministry of Health of the Russian Federation (N=54). TB, tuberculosis; XDR, extensively drug-resistant. 
$0.60 ; \mathrm{p}=0.009)$ were all associated with delayed culture conversion.

When compared to their combined use, there was evidence of a crude association between prescription of bedaquiline alone (HR: $0.12,95 \%$ CI: $0.03-0.49 ; \mathrm{p}=0.003$ ) or linezolid alone (HR: 0.21 , $95 \%$ CI: $0.06-0.69 ; \mathrm{p}=0.010)$ as part of the treatment regimen with delayed culture conversion (Table 2).

\section{Discussion}

Amongst our cohort of XDR-TB patients, we found that median time to culture conversion was 4 months using solid Lowenstein-Jensen media, and that $70 \%$ had culture converted

Table 2. Factors associated with culture conversion among adult pulmonary XDR-TB patients who started treatment between 1 January 2018 - 30 June 2019 at Federal State Budgetary Institution "National Medical Research Center of Phthisiopulmonology and Infectious Diseases" of the Ministry of Health of the Russian Federation (N=54).

\begin{tabular}{|c|c|c|c|c|c|}
\hline & $\begin{array}{c}\text { Culture } \\
\text { conversion, n }\end{array}$ & $\begin{array}{l}\text { Follow-up time } \\
\text { (person months) }\end{array}$ & $\begin{array}{l}\text { Rate per } 100 \\
\text { person-months }\end{array}$ & $\begin{array}{l}\text { Crude cause-specific } \\
\text { hazard ratio }(95 \% \mathrm{CI})\end{array}$ & p-value \\
\hline $\begin{array}{l}\text { Sex } \\
\text { Male } \\
\text { Female }\end{array}$ & $\begin{array}{l}23 \\
20\end{array}$ & $\begin{array}{l}115 \\
75\end{array}$ & $\begin{array}{l}20.0 \\
26.7\end{array}$ & $\begin{array}{c}0.78(0.42-1.45) \\
\text { ref. }\end{array}$ & 0.431 \\
\hline $\begin{array}{l}\text { Patient's age at the beginning of treatment } \\
18-34 \\
35-44 \\
45-63\end{array}$ & $\begin{array}{l}19 \\
16 \\
8\end{array}$ & $\begin{array}{l}84 \\
73 \\
34\end{array}$ & $\begin{array}{l}22.6 \\
21.9 \\
23.5\end{array}$ & $\begin{array}{l}0.98(0.50-1.92) \\
\text { ref. } \\
1.18(0.50-2.79)\end{array}$ & $\begin{array}{l}0.947 \\
0.713\end{array}$ \\
\hline $\begin{array}{l}\text { Employment } \\
\text { Employed } \\
\text { Unemployed }\end{array}$ & $\begin{array}{l}15 \\
28\end{array}$ & $\begin{array}{c}72 \\
119\end{array}$ & $\begin{array}{l}20.8 \\
23.5\end{array}$ & $\begin{array}{c}0.83(0.44-1.57) \\
\text { ref. }\end{array}$ & 0.564 \\
\hline $\begin{array}{l}\text { History of incarceration } \\
\text { Yes } \\
\text { No }\end{array}$ & $\begin{array}{c}3 \\
40\end{array}$ & $\begin{array}{c}20 \\
171\end{array}$ & $\begin{array}{l}15.0 \\
23.4\end{array}$ & $\begin{array}{c}0.62(0.19-2.04) \\
\text { ref. }\end{array}$ & 0.435 \\
\hline $\begin{array}{l}\text { Current smoking status } \\
\text { Yes } \\
\text { No }\end{array}$ & $\begin{array}{l}22 \\
20\end{array}$ & $\begin{array}{c}119 \\
69\end{array}$ & $\begin{array}{l}18.5 \\
29.0\end{array}$ & $\begin{array}{l}0.54(0.29-1.01) \\
\text { ref. }\end{array}$ & 0.053 \\
\hline $\begin{array}{l}\text { Harmful use of alcohol } \\
\text { Yes } \\
\text { No }\end{array}$ & $\begin{array}{c}4 \\
39\end{array}$ & $\begin{array}{c}31 \\
159\end{array}$ & $\begin{array}{l}12.9 \\
24.5\end{array}$ & $\begin{array}{l}0.32(0.10-1.05) \\
\text { ref. }\end{array}$ & 0.060 \\
\hline $\begin{array}{l}\text { HIV status } \\
\text { Positive } \\
\text { Negative }\end{array}$ & $\begin{array}{c}4 \\
39\end{array}$ & $\begin{array}{c}35 \\
156\end{array}$ & $\begin{array}{l}11.4 \\
25.0\end{array}$ & $\begin{array}{c}\mathbf{0 . 3 0}(\mathbf{0 . 0 9 - 0 . 9 7 )} \\
\text { ref. }\end{array}$ & 0.045 \\
\hline $\begin{array}{l}\text { Diabetes } \\
\text { Yes } \\
\text { No }\end{array}$ & $\begin{array}{c}8 \\
35\end{array}$ & $\begin{array}{c}35 \\
156\end{array}$ & $\begin{array}{l}22.9 \\
22.4\end{array}$ & $\begin{array}{c}0.92(0.42-2.01) \\
\text { ref. }\end{array}$ & 0.840 \\
\hline $\begin{array}{l}\text { Chronic hepatitis C } \\
\text { Yes } \\
\text { No }\end{array}$ & $\begin{array}{c}6 \\
37\end{array}$ & $\begin{array}{c}49 \\
142\end{array}$ & $\begin{array}{l}12.2 \\
26.1\end{array}$ & $\begin{array}{c}\mathbf{0 . 3 5}(\mathbf{0 . 1 4 - 0 . 8 9 )} \\
\text { ref. }\end{array}$ & 0.023 \\
\hline $\begin{array}{l}\text { Cavities detected on chest x-ray } \\
\text { Yes } \\
\text { No }\end{array}$ & $\begin{array}{l}30 \\
13 \\
\end{array}$ & $\begin{array}{l}140 \\
51\end{array}$ & $\begin{array}{l}21.4 \\
25.5\end{array}$ & $\begin{array}{c}0.83(0.42-1.64) \\
\text { ref. }\end{array}$ & 0.601 \\
\hline $\begin{array}{l}\text { Baseline sputum smear microscopy grade } \\
\text { Negative } \\
\text { Positive }\end{array}$ & $\begin{array}{l}18 \\
25\end{array}$ & $\begin{array}{c}54 \\
137\end{array}$ & $\begin{array}{l}33.3 \\
18.2\end{array}$ & $\begin{array}{c}\text { ref. } \\
\mathbf{0 . 3 4}(\mathbf{0 . 1 8 - 0 . 6 6 )}\end{array}$ & 0.001 \\
\hline $\begin{array}{l}\text { Previous TB treatment history } \\
\text { Previously treated } \\
\text { New }\end{array}$ & $\begin{array}{c}8 \\
35\end{array}$ & $\begin{array}{c}37 \\
154\end{array}$ & $\begin{array}{l}21.6 \\
22.7\end{array}$ & $\begin{array}{c}\text { ref. } \\
0.94(0.43-2.05)\end{array}$ & 0.869 \\
\hline $\begin{array}{l}\text { Bdq / Lzd in the treatment regimen } \\
\text { Bdq only } \\
\text { Lzd only } \\
\text { Both }\end{array}$ & $\begin{array}{c}3 \\
5 \\
35\end{array}$ & $\begin{array}{c}33 \\
37 \\
121\end{array}$ & $\begin{array}{c}9.1 \\
13.5 \\
28.9\end{array}$ & $\begin{array}{c}0.12(0.03-0.49) \\
0.21(0.06-0.69) \\
\text { ref. }\end{array}$ & $\begin{array}{l}0.003 \\
0.010\end{array}$ \\
\hline $\begin{array}{l}\text { Number of effective drugs in the treatment } \\
3 \\
4 \\
5 \\
6-8\end{array}$ & $\begin{array}{c}3 \\
7 \\
25 \\
8\end{array}$ & $\begin{array}{l}39 \\
49 \\
79 \\
24\end{array}$ & $\begin{array}{c}7.7 \\
14.3 \\
31.6 \\
33.3\end{array}$ & $\begin{array}{c}\mathbf{0 . 1 3}(\mathbf{0 . 0 3 - 0 . 6 0 )} \\
0.37(0.13-1.04) \\
1.00(0.45-2.25) \\
\text { ref. }\end{array}$ & $\begin{array}{c}\mathbf{0 . 0 0 9} \\
0.060 \\
0.992\end{array}$ \\
\hline
\end{tabular}

${ }^{\circ}$ Effective drugs are defined: MTB drug sensitivity is confirmed based on the Mycobacterium tuberculosis drug susceptibility test (DST); MTB drug sensitivity is assumed (new TB drugs or antibiotics with anti-TB activity; drugs that were not previously part of an ineffective regimen for this patient; high susceptibility of MTB strains for these drugs in patients with a similar TB history according to results of population drugresistance studies); Bdq, bedaquiline; HIV, human immunodeficiency virus; Lzd, linezolid; TB, tuberculosis; XDR, extensively drug-resistant. 
by month 6 of treatment. Comparison with other studies is challenging since our study cohort includes only XDR-TB patients who were culture-positive at baseline, while in most of others reports XDR-TB represented only small proportion of the study population. Furthermore, many studies did not report on method to follow-up sputum culture, which is essential in this analysis of time to conversion studies, since there is a strong evidence that liquid culture methods due to high sensitivity compared to solid culture methods are associated with longer times to culture conversion [21]. However, other comparable studies reported a wide range of median times to culture conversion - 1 to 6 months [2225 ] - and wide range of proportion of patients, who achieved culture conversion by month 6 of treatment - 47-97\% [22,25-27].

Previous studies have demonstrated the positive association between both bedaquiline and linezolid and culture conversion in patients with MDR/RR-TB including XDR-TB [4,22,23,26-31]. However, we additionally identified that treatment including both bedaquiline and linezolid was associated with faster sputum culture conversion when compared with treatments that only include either bedaquiline or linezolid. The effectiveness of this combination of drugs for XDR-TB has been reported by Olayanju et al. where inclusion of a combination of bedaquiline and linezolid resulted in substantially increased likelihood of favourable outcomes compared to regimens without these drugs [31]. Another single arm prospective study also demonstrated the effectiveness of the XDR-TB treatment regimen with co-administration of bedaquiline and linezolid in combination with pretomanid [32].

Our results describe an association between fewer effective drugs included in a treatment regimen and increased time to culture conversion. Compared with regimens including 6-8 effective drugs, treatment with regimens including 3 effective drugs were significantly associated with lower likelihood of culture conversion. We found moderate evidence $(\mathrm{p}=0.06)$ for a similar association between treatment with regimens including 4 effective drugs and culture conversion. More extensive treatment history and resistance to the highly effective fluoroquinolone drugs may contribute to worsen treatment responses amongst patients treated for XDR-TB. Our findings suggest that, where possible, regimens containing 5 or more effective drugs may improve time to culture conversion in XDR-TB. Other authors have also recommended including at least five anti-TB drugs with confirmed or suspected efficacy in the treatment regimen for XDR-TB [9,12,33]. This is essential for complete suppress the MTB population, taking into account the possible undetected additional drug resistance due to the limitations of existing diagnostic methods [9,34]. This approach needs to be adopted tailoring the drug selection to the drug-susceptibility testing, under the guidance of a Consilium, and monitoring strictly the potential adverse events [4].

We also found a possible evidence of an association $(\mathrm{p}=0.053)$ between smoking status and increased time to sputum conversion following adjustment for other factors. Published studies show that smoking delays culture conversion in TB patients including XDRTB $[35,36]$. Reimann et al. note that smoking leads to damage of respiratory immune-defence mechanisms, which increases susceptibility to MTB infection and delays bacterial clearance. The impact of smoking on local lung immunity is supported by the study of Lin et al. which implies that cigarette smoking influences outcomes of pulmonary but not extra-pulmonary TB [36,37].

Prevalence of HIV, hepatitis $\mathrm{C}$ and diabetes mellitus in our cohort was low, but both HIV and hepatitis C showed significant crude associations with delayed culture conversion. Results from other studies investigating the impact of HIV status on the time to culture conversion are conflicting and contradict our find- ings. Mohr et al. in their study on 853 confirmed DR-TB patients from Southern Africa revealed that HIV status did not have impact on time to sputum culture conversion and treatment success [38]. In another study by Shibabaw et al. the median time to culture conversion was significantly shorter among HIV positive patients compared to HIV negative [39]. We believe our findings may be attributed to more severe disease amongst those with HIV, presence of additional unmeasured comorbidities in this patient group and increased risk of adverse events and treatment interruption through hepatotoxicity or drug-drug interactions [40]. As treatment for hepatitis $\mathrm{C}$ has dramatically improved in recent years, serological screening for hepatitis $\mathrm{C}$ amongst those with DR-TB is becoming more common. Multiple studies have demonstrated the association between hepatitis $\mathrm{C}$ co-infection and time to culture conversion in DR-TB patients along with the increased risk of drug-related hepatotoxicity [41,42]. Since prevalence of hepatitis C and HIV are high amongst TB patients, we suggest that additional research is required into interaction of these diseases and joint management [1,43]. Similar to other studies, positive baseline sputum smear microscopy $[44,45]$ was also associated with longer time to sputum conversion. These findings are likely due to the presence of higher bacterial load and advanced parenchymal damage and less effective treatment through reduced medication exposure.

The key strength of our study is that our database included all patients enrolled into treatment at the study site within the study period and matched the inclusion criteria. For conducting and reporting observational cohort studies we adhered to the STROBE guidelines. The study settings corresponded with routine programmatic settings, ensuring representativeness of our sample. Percentage of missing data was minimal, below the generally accepted $10 \%$ threshold.

The study has several limitations. The primary limitation was the relatively low sample size that did not allow to conduct an adjusted analysis for all variables. A limitation when considering treatment effectiveness is the use of interim outcome measures. While sputum culture conversion has been shown to be predictive of treatment outcome in some studies, using end of treatment outcomes would result in more robust findings [18]. In this study, we chose to use interim outcome measures to be able to include patients who more recently started treatment, so to increase the relevance of our findings. Our study was not multicentre, limiting the generalizability of the findings. However, the inclusion the study participants from different regions of the country and the availability of general recommendations for TB treatment throughout the Russian Federation may minimize the significance of this limitation. We did not collect data on ART use among HIV co-infected patients, which limits our ability to interpret the role of HIV status in achieving culture conversion.

\section{Conclusions}

Factors delaying sputum culture conversion should be considered in the management of XDR-TB patients and the prescription of effective treatment regimens. An insufficient number of effective drugs in the treatment regimen reduces time to culture conversion, which can potentially lead to reduced treatment success. Our study outlines importance of simultaneous inclusions of bedaquiline and linezolid in the basic treatment regimen to reduce time to sputum conversion. 


\section{References}

1. WHO. Global tuberculosis report 2019. Geneva: World Health Organization; 2020. Accessed on: 2020 Jul 7. Available from: http://www.who.int/tb/publications/global_report/en/

2. WHO. Moscow Declaration to End TB. Geneva: World Health Organization; 2017. Accessed on: 2020 Jul 1. Available from: https://www.who.int/tb/Moscow_Declaration_MinisterialConf erence TB/en/

3. Lange C, Dheda K, Chesov D et al. Management of drug-resistant tuberculosis. Lancet 2019;394:953-66.

4. Migliori GB, Tiberi S, Zumla A et al. MDR/XDR-TB management of patients and contacts: Challenges facing the new decade. The 2020 clinical update by the Global Tuberculosis Network. Int J Infect Dis 2020;92:S15-25.

5. WHO. Consolidated Guidelines on Tuberculosis, Module 4: Treatment - Drug-Resistant Tuberculosis Treatment. Geneva: World Health Organization; 2020. Accessed on: 2020 Aug 5. Available from: https://www.who.int/publications/i/item/ 9789240007048

6. Pranger AD, van der Werf TS, Kosterink JGW, Alffenaar JWC. The role of fluoroquinolones in the treatment of tuberculosis in 2019. Drugs 2019;79:161-71.

7. WHO Regional Office for Europe. Tuberculosis action plan for the WHO European Region 2016-2020. Accessed on: $2020 \mathrm{Jul}$ 7. Available from: http://www.euro.who.int/en/who-we-are/governance

8. WHO. Definitions and reporting framework for tuberculosis. Geneva: World Health Organization; 2020. Accessed on: 2020 Jul 7. Available from: http://www.who.int/tb/publications/definitions/en/

9. Vasilyeva I, Balasaniantc G, Borisov S, et al. Tuberculosis in adults. Accessed on: 2020 Jul 7. Available from: http://cr.rosminzdrav.ru/\#!/recomend/943

10. WHO. WHO consolidated guidelines on drug-resistant tuberculosis treatment. Geneva: World Health Organization; 2019. Accessed on: 2020 Jul 7. Available from: http://www.who. int/tb/publications/2019/consolidated-guidelines-drug-resistant-TB-treatment/en/

11. Burmistrova I, Vaniev EV, Samoylova AG, et al. Amplification of drug resistance against the background of inadequate chemotherapy for pulmonary tuberculosis. Tuberc Lung Dis 2019;97:46-51.

12. Ahmad N, Ahuja SD, Akkerman OW, et al. Treatment correlates of successful outcomes in pulmonary multidrug-resistant tuberculosis: an individual patient data meta-analysis. Lancet 2018;392:821-34.

13. ClinicalTrials.gov. Safety and efficacy of various doses and treatment durations of linezolid plus bedaquiline and pretomanid in participants with pulmonary TB, XDR-TB, preXDR-TB or non-responsive/intolerant MDR-TB (ZeNix). Global Alliance for TB Drug Development. ID NCT03086486. Accessed on: $2020 \mathrm{Jul}$ 7. Available from: https://www.clinicaltrials.gov/ct2/show/NCT03086486.

14. ClinicalTrials.gov. Evaluating newly approved drugs for multidrug-resistant TB. Médecins Sans Frontières, France. ID NCT02754765. Accessed on: 2020 Jul 7. Available from: https://clinicaltrials.gov/ct2/show/NCT02754765

15. ClinicalTrials.gov. Pragmatic clinical trial for a more effective concise and less toxic MDR-TB treatment regimen(s). Medecins Sans Frontieres, Netherlands. ID NCT02589782. Accessed on: $2020 \mathrm{Jul}$ 7. Available from: https://clinicaltrials. gov/ct2/show/NCT02589782
16. Pontali E, Raviglione MC, Migliori GB, et al. Regimens to treat multidrug-resistant tuberculosis: Past, present and future perspectives. Eur Res Rev 2019;28:190035.

17. Bastard M, Sanchez-Padilla E, Hayrapetyan A, et al. What is the best culture conversion prognostic marker for patients treated for multidrug-resistant tuberculosis? Int J Tuberc Lung Dis 2019;23:1060-7.

18. Lu P, Liu Q, Martinez L, et al. Time to sputum culture conversion and treatment outcome of patients with multidrug-resistant tuberculosis: A prospective cohort study from urban China. Eur Res J 2017;49:1601558.

19. Kurbatova EV, Cegielski JP, Lienhardt C, et al. Sputum culture conversion as a prognostic marker for end-of-treatment outcome in patients with multidrug-resistant tuberculosis: A secondary analysis of data from two observational cohort studies. Lancet Respir Med 2015;3:201-9.

20. WHO. Technical report on critical concentrations for TB drug susceptibility testing of medicines used in the treatment of drug-resistant TB. Geneva: World Health Organization; 2018.

21. Rishi S, Sinha P, Malhotra B, Pal N. A comparative study for the detection of mycobacteria by BACTEC MGIT 960, Lowenstein Jensen media and direct AFB smear examination. Indian J Med Microbiol 2007;25:383-6.

22. Guglielmetti L, Le Dû D, Jachym M, et al. Compassionate use of bedaquiline for the treatment of multidrug-resistant and extensively drug-resistant tuberculosis: Interim analysis of a French cohort. Clin Infect Dis 2015;60:188-94.

23. Borisov SE, Dheda K, Enwerem M, et al. Effectiveness and safety of bedaquilinecontaining regimens in the treatment of MDR- and XDR-TB: A multicentre study. Eur Respir J 2017;49: 1700387.

24. Liu Q, Lu P, Martinez L, et al. Factors affecting time to sputum culture conversion and treatment outcome of patients with multidrug-resistant tuberculosis in China. BMC Infect Dis 2018;18:114.

25. Qazi F, Khan U, Khowaja S, et al. Predictors of delayed culture conversion in patients treated for multidrug-resistant tuberculosis in Pakistan. Int J Tuberc Lung Dis 2011;15:1556-9.

26. Gao M, Gao J, Xie L et al. Early outcome and safety of bedaquiline-containing regimens for treatment of MDR- and XDR-TB in China: a multicentre study. Clin Microbiol Infect 2020. doi: 10.1016/j.cmi.2020.06.004

27. Salhotra VS, Sachdeva KS, Kshirsagar N, et al. Effectiveness and safety of bedaquiline under conditional access program for treatment of drug-resistant tuberculosis in India: An interim analysis. Indian J Tuberc 2020;67:29-37.

28. Lifan Z, Sainan B, Feng S, et al. Linezolid for the treatment of extensively drug-resistant tuberculosis: A systematic review and meta-analysis. Int J Tuberc Lung Dis 2019;23:1293-307.

29. Sarin R, Singla N, Vohra V et al. Initial experience of bedaquiline implementation under the National TB Programme at NITRD, Delhi, India. Indian J Tuberc 2019;66:209-13.

30. Pym AS, Diacon AH, Tang SJ, et al. Bedaquiline in the treatment of multidrug- and extensively drugresistant tuberculosis. Eur Respir J 2016;47:564-74.

31. Olayanju O, Limberis J, Esmail A, et al. Long-term bedaquiline-related treatment outcomes in patients with extensively drug-resistant tuberculosis from South Africa. Eur Respir J 2018;51:1800544.

32. Conradie F, Diacon AH, Ngubane N, et al. Treatment of highly drug-resistant pulmonary tuberculosis. $N$ Engl J Med 2020;382:893-902. 
33. Matteelli A, Roggi A, Carvalho ACC. Extensively drug-resistant tuberculosis: Epidemiology and management. Clin Epidemiol 2014;6:111-8.

34. Migliori GB, Sotgiu G, Gandhi NR, et al. Drug resistance beyond extensively drugresistant tuberculosis: Individual patient data meta-analysis. Eur Respir J 2013;42:169-79.

35. Wang EY, Arrazola RA, Mathema B, et al. The impact of smoking on tuberculosis treatment outcomes: a meta-analysis. Int J Tuberc Lung Dis 2020;24:170-5.

36. Reimann M, Schaub D, Kalsdorf B, et al. Cigarette smoking and culture conversion in patients with susceptible and M/XDR-TB. Int J Tuberc Lung Dis 2019;23:93-8.

37. Lin H-H, Ezzati M, Murray M. Tobacco smoke, indoor air pollution and tuberculosis: A systematic review and meta-analysis. PLoS Med 2007;4:e20.

38. Mohr E, Cox V, Wilkinson L, et al. Programmatic treatment outcomes in HIV-infected and uninfected drug-resistant TB patients in Khayelitsha, South Africa. Trans R Soc Trop Med Hyg 2015; 109:425-32.

39. Shibabaw A, Gelaw B, Wang S-H, Tessema B. Time to sputum smear and culture conversions in multidrug resistant tuberculosis at University of Gondar Hospital, Northwest Ethiopia. PLoS One 2018;13:e0198080.

40. Lomtadze N, Kupreishvili L, Salakaia A, et al. Hepatitis C virus co-infection increases the risk of anti-tuberculosis drug- induced hepatotoxicity among patients with pulmonary tuberculosis. PLoS One 2013;8:e83892.

41. El-Serag HB, Anand B, Richardson P, Rabeneck L. Association between hepatitis $\mathrm{C}$ infection and other infectious diseases: A case for targeted screening? Am J Gastroenterol 2003;98:167-74.

42. Behzadifar M, Heydarvand S, Behzadifar M, Bragazzi NL. Prevalence of hepatitis $\mathrm{C}$ virus in tuberculosis patients: A systematic review and meta-analysis. Ethiop J Health Sci 2019;29:945-56.

43. Agha M, El-Mahalawy I, Seleem H, Helwa M. Prevalence of hepatitis $\mathrm{C}$ virus in patients with tuberculosis and its impact in the incidence of anti-tuberculosis drugs induced hepatotoxicity. Eur Res J 2015;PA2983.

44. Kurbatova E, Gammino VM, Bayona J, et al. Predictors of sputum culture conversion among patients treated for multidrug-resistant tuberculosis. Int $\mathrm{J}$ Tuberc Lung Dis 2012;16:1335-43.

45. Parmar MM, Sachdeva KS, Dewan PK, et al. Unacceptable treatment outcomes and associated factors among India's initial cohorts of multidrug-resistant tuberculosis (MDR-TB) patients under the revised national TB control programme (2007-2011): Evidence leading to policy enhancement. PLoS One 2018;13:e0193903. 